\title{
Direct photon cross sections in proton-proton and antiproton-proton interactions at $\sqrt{s}=24.3 \mathrm{GeV}$
}

\author{
UA6 Collaboration \\ CERN-Lausanne-Michigan-Rockefeller
}

G. Ballocchi a,c,13, R.E. Breedon ${ }^{\mathrm{d}, 10}{ }^{\text {, L. Camilleri }}{ }^{\mathrm{a}}$, C. Comtat b,2, R.L. Cool d,16, P.T. Cox ${ }^{\mathrm{d}, \mathrm{a}, \mathrm{b}, 10 \text {, }}$ P. Cushman ${ }^{\mathrm{d}, e, 9}$, L. Dick ${ }^{a}$, E.C. Dukes ${ }^{\mathrm{c}, 3}$, P. Giacomelli d,12, D.B. Hubbard ${ }^{c, 14}$, J.B. Jeanneret ${ }^{a}$, C. Joseph ${ }^{\text {b,1, }}$, W. Kubischta ${ }^{\text {a }}$, C. Morel ${ }^{\text {b }}$, M.-C. Nguyen ${ }^{\text {b }}$, P. Oberson b,4, O.E. Overseth ${ }^{c}$, J.L. Pagès ${ }^{b, 5}$, J.P. Perroud ${ }^{b}$, D. Rüegger ${ }^{b, 6}$, R.W. Rusack d,9, V.M. Singh e,15, G.R. Snow d,c,7, G. Sozzi b,8, L. Studer ${ }^{\text {b }}$, M.-T. Tran ${ }^{\text {b }}$, A. Vacchi ${ }^{\mathrm{d}, 11}$, G. Valenti ${ }^{\mathrm{c}, 12}$, M. Werlen ${ }^{\mathrm{b}}$

${ }^{a}$ CERN, CH-1211 Geneva 23, Switzerland

${ }^{\mathrm{b}}$ Université de Lausanne, CH-1015 Lausanne, Switzerland

${ }^{\mathrm{c}}$ University of Michigan, Ann Arbor MI 48109, USA

${ }^{\mathrm{d}}$ Rockefeller University, New York, NY 10021, USA

e Yale University, New Haven, CT 06511, USA

\begin{abstract}
We report results on inclusive direct photon $(\gamma), \pi_{0}$, and $\eta$ production in both pp and $\overline{\mathrm{p}} \mathrm{p}$ interactions at $\sqrt{s}=24.3 \mathrm{GeV}$ in the transverse momentum range $4.1 \leq p_{T} \leq 7.7$ $\mathrm{GeV} / c$ and rapidity range $-0.1 \leq y \leq 0.9$. The data were collected between 1988 and 1990 by the UA6 experiment at CERN, which employed an internal $\mathrm{H}_{2}$ gas jet target in the SpppS collider. The inclusive direct photon cross sections and the cross section difference $\sigma(\bar{p} p)-\sigma(p p)$ expressed as functions of $p_{T}(\gamma)$ are compared with next-to-leading order QCD predictions.
\end{abstract}




\section{Introduction}

The UA6 experiment was designed to study high $p_{T}$ phenomena and small angle elastic scattering in proton-proton and antiproton-proton interactions at $\sqrt{s}=24.3 \mathrm{GeV}$. The main emphasis of the experiment was the study of high $p_{T}$ direct photons. The production of direct photons in pp interactions, which to first order proceeds mainly through the quark-gluon scattering process, depends on the gluon distribution in the proton, and has been well-explored in our energy domain by several experiments [1]. Experiment UA6 allowed a precise measurement of both the pp and $\bar{p} p$ cross sections. The contribution of the quark-gluon process enters in both channels and cancels in the difference of the cross sections $\sigma(\bar{p} p \rightarrow \gamma X)-\sigma(p p \rightarrow \gamma X)$. This difference is then sensitive to the $\overline{\mathrm{q}} \mathrm{q}$ annihilation process. First results of this experiment based on data collected in 1985 and 1986 have already been published [2,3]. In this paper we present results from measurements performed in 1988 for pp, with an integrated luminosity of $3.90 \mathrm{pb}^{-1}$, and in 1989 and 1990 for $\bar{p}$ p with an integrated luminosity of $4.75 \mathrm{pb}^{-1}$ (an order of magnitude increase over our earlier $\bar{p} \mathrm{p}$ results). Both data samples cover a range in rapidity $-0.1 \leq y \leq 0.9$, and a range in transverse momentum $4.1 \leq p_{T} \leq 7.7 \mathrm{GeV} / c$, which corresponds to an $x_{T}\left(=2 p_{T} / \sqrt{s}\right)$ range $0.34 \leq x_{T} \leq 0.63$.

\section{Apparatus}

The apparatus of experiment UA6 was located in a long straight section of the CERN S̄ppS collider between 1984 and 1990. It used a molecular hydrogen cluster

1 Corresponding author

2 Present address: University of Pittsburgh, Pittsburgh PA 15260, USA

3 Present address: University of Virginia, Charlottesville VA 22901, USA

4 Present address: Reuter Agency, Geneva, Switzerland

5 Present address: IBM, Geneva, Switzerland

6 Present address: École Technique Supérieure, Yverdon, Switzerland

7 Present address: University of Nebraska, Lincoln NE 68588, USA

8 Present address: Lotus Development (Suisse), Geneva, Switzerland

9 Present address: University of Minnesota, Minneapolis, MN 55455,USA

${ }^{10}$ Present address: University of California, Davis, CA 95616 ,USA

${ }^{11}$ Present address: INFN, Trieste, I-34127, Italy

${ }^{12}$ Present address: INFN, Bologna, I-40126, Italy

${ }^{13}$ Present address: Olsen \& Associates, Zurich, Switzerland

${ }^{14}$ Present address: Ericson Telecom AB, 14980 Nynäshamn, Sweeden

${ }^{15}$ Present address: Bell Laboratories, New Jersey, USA

${ }^{16}$ Deceased 
jet [4] as an internal target for both the proton and the antiproton circulating beams. The jet consisted of a vertical stream of hydrogen clusters. The dimensions of the interaction volume were defined along the beam by the length of the jet ( 8 $\mathrm{mm})$, vertically by the height of the $\mathrm{S} \overline{\mathrm{p} p S}$ beam $(<1 \mathrm{~mm})$ and horizontally by the overlap of the jet and the beam (each $3 \mathrm{~mm}$ wide). The maximum density achievable by the jet was $4 \times 10^{14}$ protons $/ \mathrm{cm}^{3}$.

Two different luminosity monitors were used. A relative luminosity monitor consisting of a set of scintillation counters provided a coincidence rate proportional to the rate of interactions. The absolute luminosity was measured by detecting recoil protons from elastic scattering using two solid state detectors placed near $90^{\circ}$ in the laboratory. From the number of detected protons and the known elastic cross section in the range $2 \cdot 10^{-3} \leq t \leq 12 \cdot 10^{-3}(\mathrm{GeV} / \mathrm{c})^{2}$, the absolute luminosity over a given period was deduced and used to calibrate the relative luminosity device.

In order to keep the probability of multiple interactions occurring during one bunch crossing at a negligible level, the density of the jet was adjusted according to the circulating beam intensity so that the instantaneous luminosity did not exceed $2 \times 10^{30} \mathrm{~cm}^{-2} \mathrm{~s}^{-1}$.

The detection apparatus (fig. 1) consisted of two arms situated above and below the beam pipe. Each arm covered 20 to $100 \mathrm{mrad}$ in polar angle and 1.22 rad in azimuth, corresponding to $1.8 \mathrm{sr}$ in the centre of mass system. The essential components of each arm for this measurement were the electromagnetic calorimeter and the set of five proportional chambers for charged particle tracking. Each calorimeter [5] consisted of 28 lead plates, each 0.7 radiation length $\left(X_{0}\right)$ thick, interleaved with alternating layers of horizontal and vertical proportional tubes of rectangular section with $1 \mathrm{~cm}$ transverse dimension and 0.5 $\mathrm{cm}$ depth. Each calorimeter was divided in depth into three modules A, B and C. The front module, A, had a thickness of $6.64 X_{0}$, the other two were each $8.3 X_{0}$ thick. The analog signals of the tubes directly behind one another were summed within each module. Another module (CalUp) consisting of three layers of proportional tubes (each of $0.5 \mathrm{~cm}$ transverse dimension), one layer of vertical tubes and the other layers with tubes making angles of $+30^{\circ}$ and $-30^{\circ}$ with the vertical, was installed between modules $\mathrm{A}$ and $\mathrm{B}$ in order to resolve reconstruction ambiguities. The calorimeter was tested in an electron beam and was found to have a linear response between 2 and $140 \mathrm{GeV}$ with an energy resolution $\sigma(E) / E=0.017 \oplus 0.33 / \sqrt{E}(E$ in $\mathrm{GeV})$.

The energy scale of the calorimeter was established using the mass peak of $\pi^{0}$ s reconstructed from photon pairs. On-line the energy scale was kept approximately constant by adjusting the high voltage of the proportional tubes every hour. Offline the energy scale was determined more precisely using both $\pi^{0}$ and $\eta$ mass 
peaks, and cross-checked using the momenta of identified electrons.

During the data-taking of 1988 and 1989, a two-level trigger was used. Level 1 required a minimum energy deposition in a set of horizontal scintillators located between modules A and B of each calorimeter. The events selected by this pretrigger were then analysed by a hardware processor which:

i) read the analog sum of groups of 6 adjacent calorimeter channels of the first and second modules into fast ADCs;

ii) grouped the energy in the calorimeters into overlapping bands of 12 channels in both vertical and horizontal views;

iii) summed each horizontal band with each vertical band;

iv) converted the energy in each of the sums into $p_{T}$, assuming that the energy was deposited at the geometrical intersection of the two bands;

v) accepted the event if this $p_{T}$ exceeded the minimum $p_{T}$ required, typically 3 $\mathrm{GeV} / \mathrm{c}$.

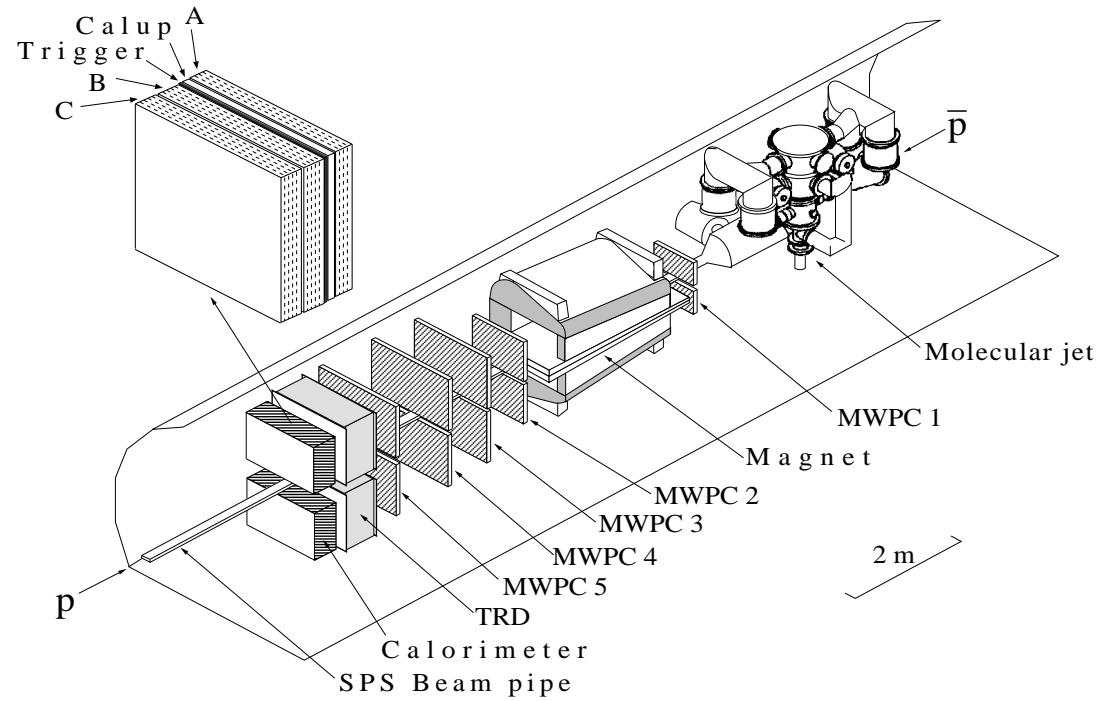

Fig. 1. The UA6 detector in the CERN S̄ppS collider oriented to view $\bar{p} p$ interactions. Insert : Structure of the calorimeter.

In 1990 a one-level trigger was used, based on the pulse height recorded in a scintillator hodoscope located between modules A and B [6]. The hodoscope consisted of sets of scintillator tiles arranged along arcs centered on the beam. Signals from any combination of four adjacent tiles were added and discriminated with a threshold that varied according to the position of the group of tiles so as to correspond to a transverse momentum of $3.3 \mathrm{GeV} / \mathrm{c}$. Because it was based on the sampling of the shower in a single scintillator layer situated behind the first module of the calorimeter, this trigger was biased against late-showering photons compared to the trigger used in 1988 and 1989, which sampled the showers over the full 15 radiation lengths of modules $\mathrm{A}$ and $\mathrm{B}$. This bias was estimated by 
comparing the energy distributions recorded in module A in 1989 and 1990. The appropriate correction factors were introduced in the Monte Carlo simulation which was used to compute the experimental acceptance.

\section{Analysis}

A clustering algorithm, which improved over that used in our earlier publication [2], was developed to analyse the calorimeter data. The procedure was as follows:

- For each "view" (horizontal tubes, H, or vertical tubes, V), clusters of adjacent tubes each containing more than $125 \mathrm{MeV}$ were formed. In modules $\mathrm{B}$ and $\mathrm{C}$, only clusters of more than one tube were considered further.

- Clusters were split into "peaks" when more than one maximum was found in a cluster.

- Peaks were associated in depth in modules A, B and C. In general, electromagnetic showers were broader in module B than in module A. Therefore, when two adjacent peaks in the A module were associated with a single broad cluster in module $\mathrm{B}$, the latter was split according to the location of the extrapolated position of A peaks onto B.

- Peaks in $\mathrm{H}$ and $\mathrm{V}$ views were then associated to form reconstructed showers in the calorimeters. For more than one particle traversing the calorimeter, some $\mathrm{H} / \mathrm{V}$ matching ambiguities were resolved using the inclined tubes in CalUp. Remaining ambiguities were resolved using the fact that for each particle the energy deposited in the $\mathrm{H}$ and $\mathrm{V}$ tubes should be approximately equal. A $\chi^{2}$ method [2] was used to select the most probable $\mathrm{H}-\mathrm{V}$ combination.

- Showers in the calorimeters for which no charged particle, reconstructed with the chambers, extrapolated to within $1.5 \mathrm{~cm}$ of the shower center were assumed to be photons.

- Only photons well within the magnet acceptance were used. Photons in the same arm were combined in pairs and their invariant mass computed assuming they originated from the target.

- Photons which did not combine to form a $\pi^{0}$, defined as $80 \leq m_{\gamma \gamma} \leq 200 \mathrm{MeV} / c^{2}$, were considered as direct photon candidates.

A detailed Monte-Carlo simulation of the full apparatus was developed. The simulation of the shower development in the calorimeter was performed using a bank of electron showers collected in a test beam and slightly modified to account for the differences between photon and electron showers. These modified showers were found to be consistent with GEANT [7] shower simulations. The Monte Carlo was used to evaluate the acceptance of the detector, and to estimate the trigger efficiency. It was also used to evaluate the contamination of the direct photon sample. This contamination arises from those $\pi^{0}$ 's for which the photon showers merge into a single cluster, and from asymmetric $\pi^{0}$ decays in which one 
photon is not observed. The small contribution from other neutral meson decays was also taken into account.

\section{Results}

The invariant cross sections for $\pi^{0}$ production are shown as a function of transverse momentum in figure $2 \mathrm{a}$ and as a function of rapidity in figure $2 \mathrm{~b}$. The ratio of the $\bar{p} p$ to the pp cross sections is shown in figure 2c. When the cross sections are integrated over $p_{T}$ and $y$ this ratio is $0.98 \pm 0.02 \pm 0.10$ where the first error is the statistical uncertainty and the second is the overall systematic uncertainty estimated as described at the end of this section.

We have also extracted the ratios of cross sections for $\eta$ to $\pi^{0}$ production in $\bar{p} p$ and pp. This ratio, shown as a function of $p_{T}$ in figure $3 \mathrm{a}$ and as a function of $y$ in figure $3 \mathrm{~b}$, can be seen to be very similar in the two types of interactions.

The invariant cross sections for direct photon production as a function of $p_{T}$ and $y$ are shown in figure $4 \mathrm{a}$ and figure $4 \mathrm{~b}$. The much larger $\bar{p}$ p invariant cross section demonstrates the importance of the valence $\bar{q} q$ annihilation. This excess is seen to occur in the central rapidity region, again in accordance with the expectation of the annihilation of a quark and an antiquark with similar $x$ values. The direct photon cross sections presented here are compatible with the results presented in our previous publication [2] which were based on earlier data corresponding to much lower luminosity.

The error bars in the figures represent statistical errors only. Systematic uncertainties on the photon cross sections which could arise at various stages of the data collection and analysis are as follows:

- The largest source of systematic uncertainty is related to the determination of the absolute energy scale of the calorimeter. From the consistency of the $\pi^{0}$ and $\eta$ masses we find that this scale is known within $0.5 \%$ and $0.7 \%$ over the measurement periods for $\mathrm{pp}$ and $\overline{\mathrm{p}} \mathrm{p}$ respectively. A change of $0.5 \%$ in the energy scale results in a change of $5 \%$ in the pp cross section and a change of $0.7 \%$ results in a change of $6 \%$ in the $\bar{p} p$ cross section. Accordingly we attribute a systematic uncertainty of $6 \%$ to both $\bar{p} p$ and pp cross sections due to knowledge of the energy scale.

- The luminosity is uncertain to within $\pm 3.3 \%$. This uncertainty is due to the knowledge of the solid angle subtended by the sensitive area of the silicon detectors used to monitor elastic recoil protons and to changes in precise running conditions over the whole data-taking period, including changes in electronics and the detectors themselves, as well as the scintillation counters used to monitor the luminosity over short time-scales. 


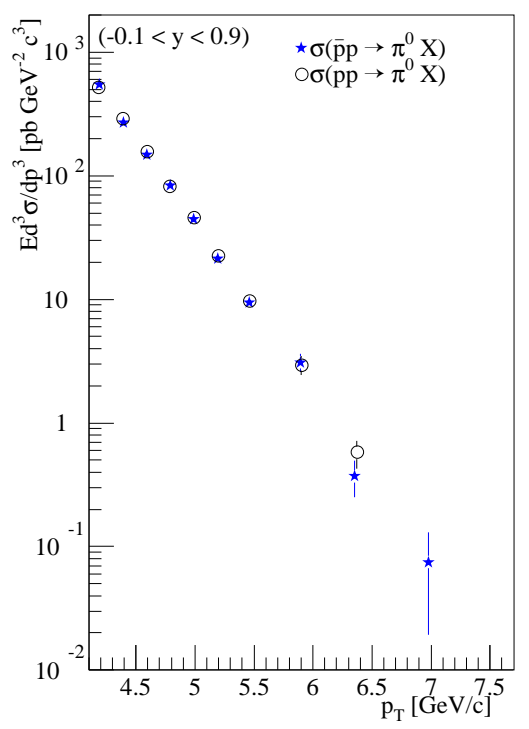

(a)

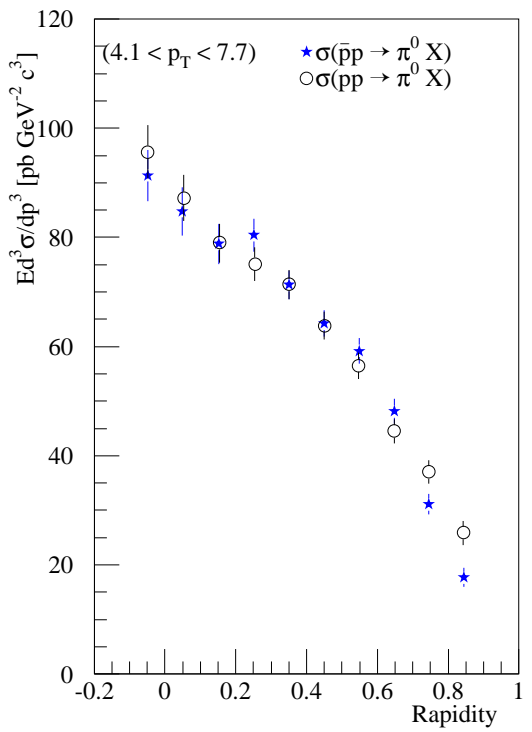

(b)
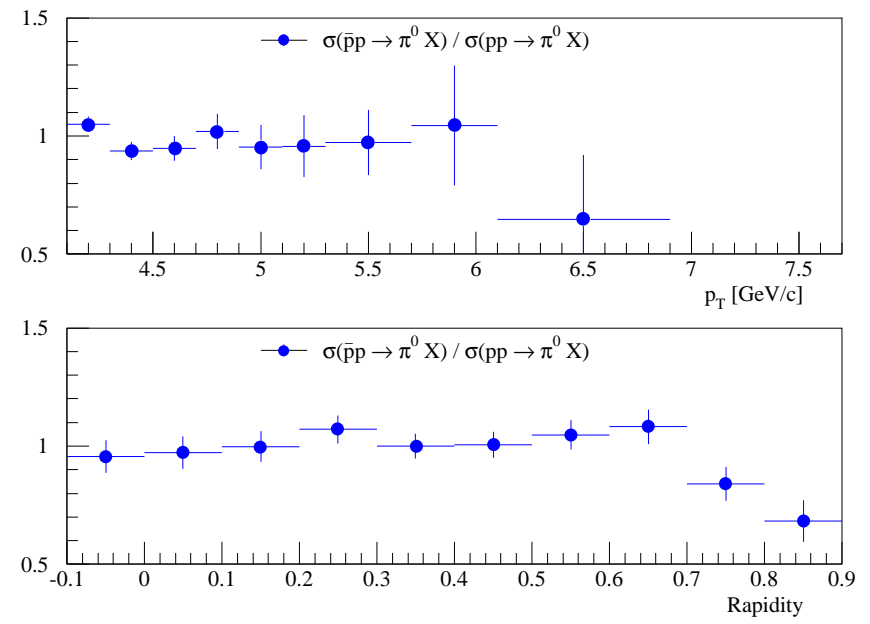

(c)

Fig. 2. $\pi^{0}$ invariant cross sections in $\bar{p} p$ and $\mathrm{pp}$ as a function of (a) transverse momentum, and (b) rapidity and (c) their ratios ( $\overline{\mathrm{p}} / \mathrm{pp})$ as a function of transverse momentum and rapidity.

- The background under the $\pi^{0}$ mass peak is assumed to be represented by a smooth function over the mass region 0 to $1 \mathrm{GeV} / c^{2}$. The value of this background is determined by fitting this mass region to a sum of the background function plus Gaussians due to $\pi^{0}$ and $\eta$. The precise shape of the background function affects the estimated number of $\pi^{0}$ candidates and hence also the estimated contamination of the direct photon sample by photons from unidentified 
$\pi^{0}$ decays. Extreme changes in this background, while still compatible with the observed mass spectrum, modify the direct photon cross section by at most $\pm 1 \%$. - During some of the $\bar{p}$ data collection periods, the proportional chambers were not fully efficient and hence the number of charged particles was underestimated. This resulted in an uncertainty on the direct photon $\bar{p}$ p cross section of $-0.5 \%$.

- Neutral hadrons are not explicitly identified and hence may contaminate the direct photon sample. Based on PYTHIA [8] studies, we estimate a systematic uncertainty of $-1 \%$ due to the neutral hadrons background.

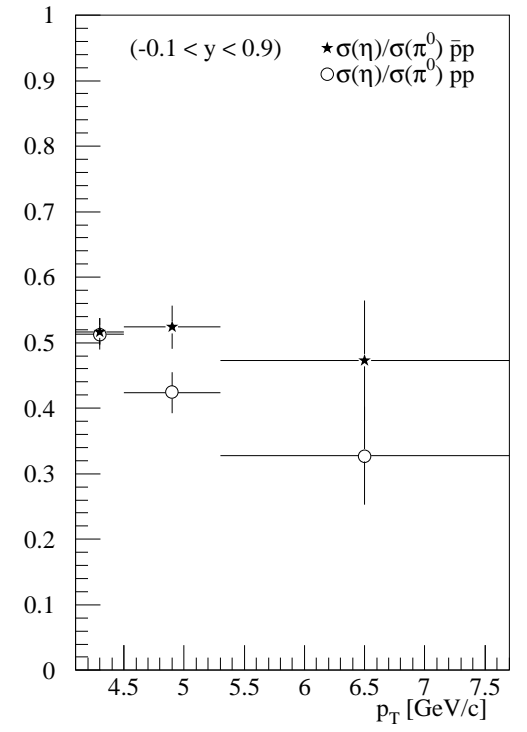

(a)

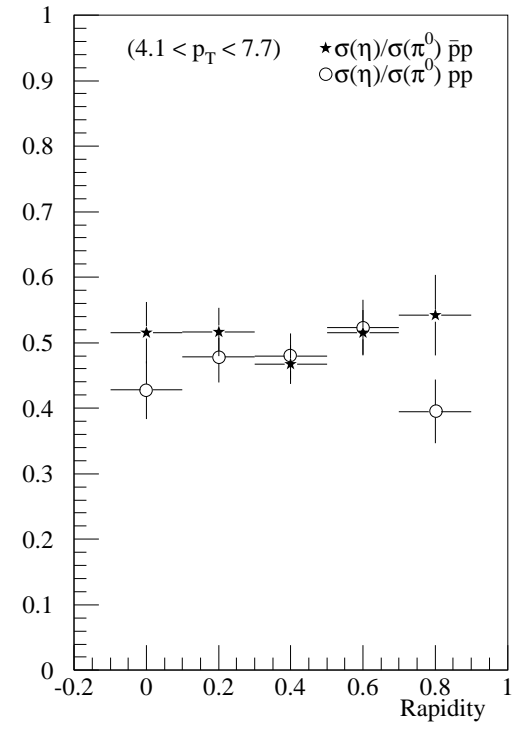

(b)

Fig. 3. The ratio of $\eta$ to $\pi^{0}$ invariant cross sections in $\bar{p} p$ and $p p$ as a function of (a) transverse momentum and (b) rapidity

- In about $2 \%$ of the events the calorimeter channels were affected by coherent pick-up noise of less than $100 \mathrm{MeV}$. This was corrected on an event by event basis by measuring the effect on channels far from the clusters. The ADC's used to measure these signals used automatic pedestal subtraction, so that only positive pick-up was seen. To evaluate the effect of possible negative, but unseen, pick-up, the data were reanalyzed with subtraction of twice the normally-observed positive pick-up. The change in photon cross section, $+2.1 \%$, is taken to represent the systematic uncertainty due to this noise.

- The shower reconstruction algorithm depends on two adjustable parameters: a cut on the minimum energy fraction in module $\mathrm{A}$ attributed to the peak, and the minimum distance between clusters in the same module before the clusters are merged into one. These parameters were tuned by detailed studies of real and Monte Carlo data. To evaluate the uncertainty incurred by a change in these parameters, they have been independently varied until the comparison between 
data and Monte Carlo appears unacceptable. The changes induced in the photon cross section at these points have been combined quadratically to give an overall change of $4.2 \%$. We attribute this value to the systematic uncertainty in the shower reconstruction procedure.

- The Monte Carlo, used for determination of the acceptance and trigger efficiency, contributes several more sources of potential systematic uncertainty:

- The use of a one-particle Monte Carlo rather than a full-event simulation has been studied by generating direct photon events using PYTHIA. These events were then analyzed using either all particles, or just the highest $p_{T}$ particle. The resulting direct photon cross sections differ by $1.2 \%$. This was taken as the systematic uncertainty due to potential event complexity.

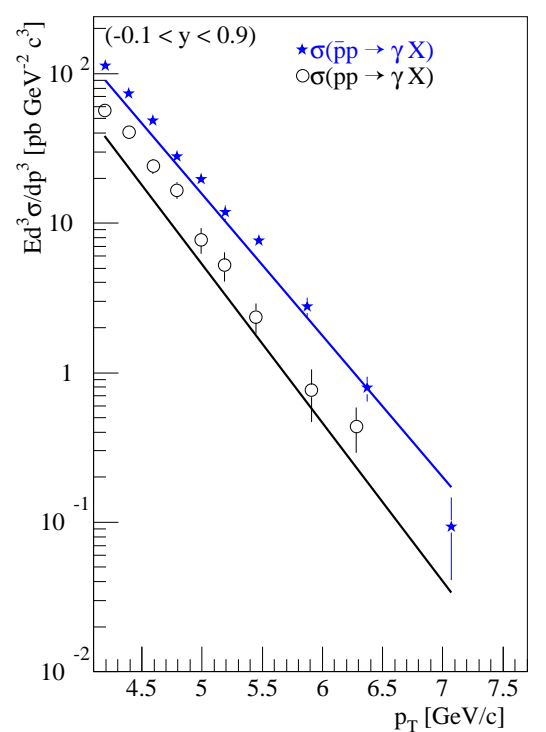

(a)

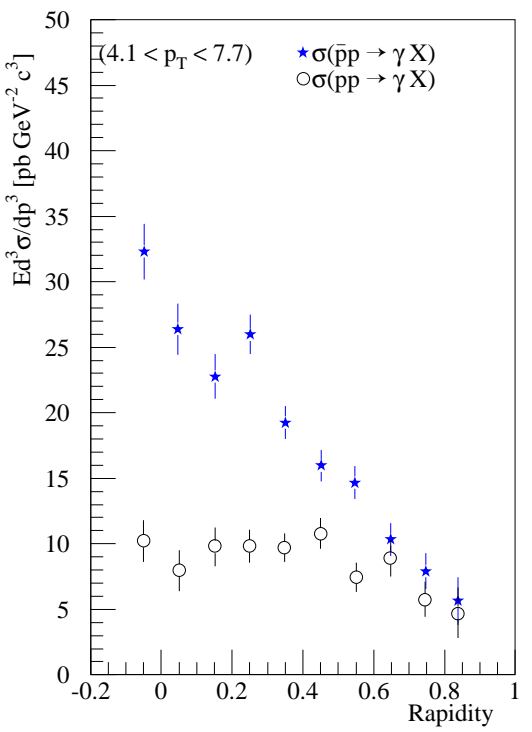

(b)

Fig. 4. Direct photon invariant cross sections in $\bar{p} p$ and $p p$ as a function of (a) transverse momentum, and (b) rapidity. The solid line are the QCD predictions referred in the text.

- The one-particle Monte Carlo used a generator of the form $(1-x)^{m} / p_{T}^{2 n}$ where $\mathrm{m}$ and $\mathrm{n}$ were determined by an iterative procedure involving the comparison of the generated distributions in $p_{T}$ and y to the acceptance-corrected data distributions. Values were determined for $\mathrm{pp}$ and $\overline{\mathrm{p}} \mathrm{p}$ separately, and for $\pi^{0}$ and gamma separately in each case. A difference of one standard deviation in these parameters induced a change in the photon cross section of $2 \%$. We consider this the systematic uncertainty associated with the form of the generator used.

- The energy resolution of the calorimeter was determined to be $(0.017 \pm$ 
$0.001) \oplus(0.329 \pm 0.002) / \sqrt{E}$ by test-beam measurements, measurements of the width of the $\eta$ mass peak and comparison of the $\mathrm{H}$ and $\mathrm{V}$ energy distributions. An overall change of $0.02 / \sqrt{E}$ led to Monte Carlo distributions which were no longer compatible with those of the data, and a change in the photon cross section of $1.8 \%$.

- The Monte Carlo used showers from an electron shower bank, slightly modified to account for the differences in longitudinal development of electron and photon showers. This modification involved an adjustable parameter, which was tuned by comparing the longitudinal distributions of the Monte Carlo showers to those of photons from reconstructed $\pi^{0}$ s. The maximum change in this parameter, while maintaining agreement between the data and Monte Carlo, induced a change in the direct photon cross section of $5.6 \%$.

Table 1

The average calculated over the interval of rapidity $[-0.1<y<0.9]$ invariant cross sections $\sigma(\overline{\mathrm{p}} \mathrm{p} \rightarrow \gamma \mathrm{X})$ and $\sigma(\mathrm{pp} \rightarrow \gamma \mathrm{X})$, and the difference $\sigma(\overline{\mathrm{p}} \mathrm{p} \rightarrow \gamma \mathrm{X})-\sigma(\mathrm{pp} \rightarrow \gamma \mathrm{X})$ as a function of $p_{T}$. The statistical errors are also given. The estimated systematic errors on the individual cross sections and on the difference are quoted in the text.

\begin{tabular}{|c|c|c|c|c|c|c|c|}
\hline $\begin{array}{c}p_{T} \text { range } \\
\mathrm{GeV} / \mathrm{c}\end{array}$ & $\begin{array}{l}<p_{T}> \\
\mathrm{GeV} / \mathrm{c}\end{array}$ & \multicolumn{2}{|c|}{$\begin{array}{c}<\sigma(\bar{p} p \rightarrow \gamma X)> \\
\mathrm{pb} \mathrm{c}^{3} / \mathrm{GeV}^{2}\end{array}$} & \multicolumn{2}{|c|}{$\begin{array}{c}<\sigma(p p \rightarrow \gamma X)> \\
\mathrm{pb} \mathrm{c}^{3} / \mathrm{GeV}^{2}\end{array}$} & \multicolumn{2}{|c|}{$\begin{array}{c}\text { Difference } \\
\mathrm{pb} \mathrm{c}^{3} / \mathrm{GeV}^{2}\end{array}$} \\
\hline $4.1-4.3$ & 4.19 & 112.7 & \pm 5.3 & 56.3 & \pm 4.9 & 56.4 & \pm 7.2 \\
\hline $4.3-4.5$ & 4.39 & 73.9 & \pm 4.0 & 40.3 & \pm 3.7 & 33.6 & \pm 5.5 \\
\hline $4.5-4.7$ & 4.59 & 48.7 & \pm 3.1 & 24.1 & \pm 2.7 & 24.6 & \pm 4.1 \\
\hline $4.7-4.9$ & 4.79 & 28.0 & \pm 2.3 & 16.7 & \pm 2.2 & 11.3 & \pm 3.1 \\
\hline $4.9-5.1$ & 4.99 & 19.6 & \pm 1.8 & 7.7 & \pm 1.5 & 11.8 & \pm 2.3 \\
\hline $5.1-5.3$ & 5.19 & 11.8 & \pm 1.4 & 5.2 & \pm 1.2 & 6.6 & \pm 1.8 \\
\hline $5.3-5.7$ & 5.46 & 7.63 & \pm 0.72 & 2.37 & \pm 0.53 & 5.26 & \pm 0.90 \\
\hline $5.7-6.1$ & 5.89 & 2.77 & \pm 0.41 & 0.76 & \pm 0.29 & 2.01 & \pm 0.50 \\
\hline $6.1-6.9$ & 6.32 & 0.79 & \pm 0.15 & 0.44 & \pm 0.15 & 0.35 & \pm 0.21 \\
\hline $6.9-7.7$ & 7.07 & 0.093 & \pm 0.052 & 0.00 & $\begin{array}{l}+0.03 \\
{ }_{-0.00}\end{array}$ & 0.093 & $\begin{array}{l}+0.060 \\
{ }_{-0.052}\end{array}$ \\
\hline
\end{tabular}

All sources of systematic uncertainty discussed above are independent, so we have evaluated the overall error as their quadratic sum. This leads to a global systematic uncertainties of $\pm 11 \%$ on the direct photon cross section for both pp and $\bar{p} p$. The systematic error on the difference of these cross sections $\sigma(\bar{p} p)-\sigma(p p)$ is $\pm 13 \%$. Finally the systematic uncertainty on the $\pi^{0}$ cross section amounts to $\pm 10 \%$ in both $\bar{p}$ and pp interactions. Excluding sources of systematic uncer- 
tainties which would affect the $\bar{p}$ p and the pp cross sections in the same way, this leads to a systematic uncertainty of $\pm 10 \%$ on the ratio of these cross sections.

In figure 4a we also show, as an example, theoretical predictions from a next to leading order QCD calculation [9]. This calculation, which includes a bremsstrahlung contribution $[10,11]$, has been performed with the renormalization scale $\mu$, the factorization scale $\mathrm{M}$, and the fragmentation scale $M_{F}$ such that $\mu=M=M_{F}=$ $p_{T} / 2$, and used the ABFOW structure functions [12]. In this calculation, the QCD scale parameter $\Lambda_{\overline{\mathrm{MS}}}^{(4)}$ was equal to $230 \mathrm{MeV}$ and the gluon distribution function was parameterized as $x G\left(x, Q_{o}^{2}\right)=A(1-x)^{\eta}$ at $Q_{o}^{2}=2 \mathrm{GeV}^{2}$ with $\eta=4.0$. This calculation, which is not a fit of these parameters to our data, already reproduces our measured cross sections reasonably well. The extraction of these parameters from these data will form the subject of a subsequent letter.

Finally, we show the difference of the $\gamma$ cross sections in $\bar{p}$ p and pp as function of $p_{T}$ in figure 5a. This figure also shows the result from our earlier publication [2]; the improvement in precision is evident. In this difference the contribution from

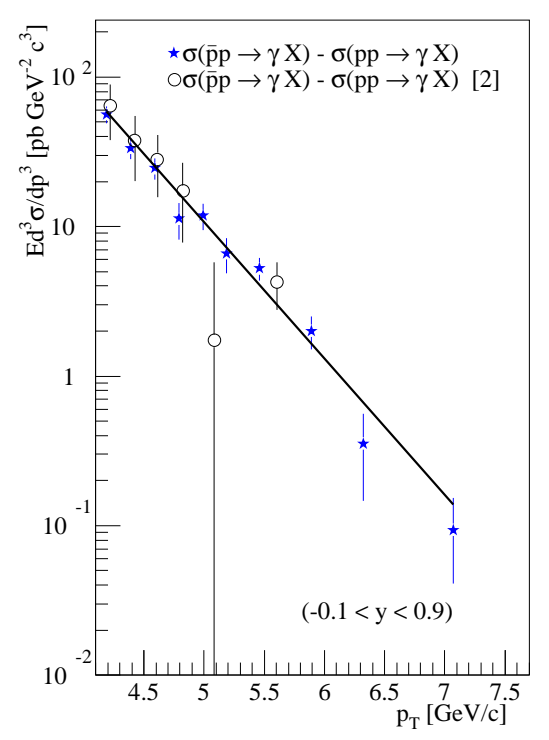

(a)

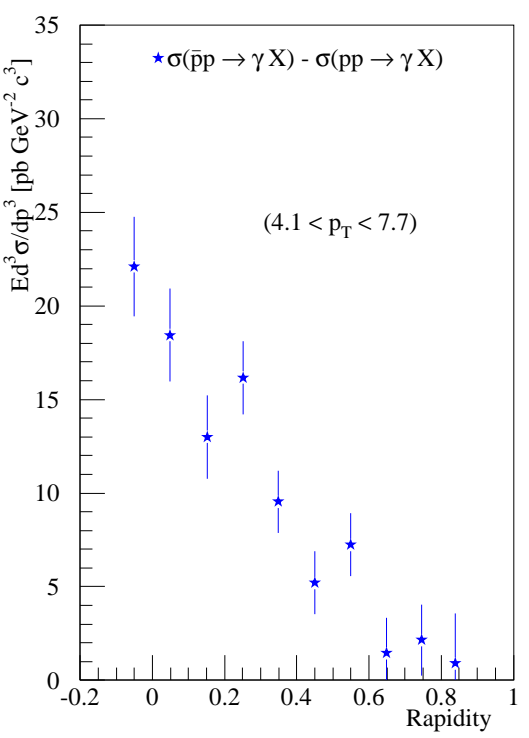

(b)

Fig. 5. Difference of direct photon cross sections in $\bar{p} p$ and pp as a function of (a) transverse momentum, and (b) rapidity.

quark-gluon scattering almost vanishes and the result, which depends essentially on only the quark-antiquark annihilation, provides a measurement of the strong coupling constant $\alpha_{s}$. The solid line in figure 5 a represents the result of a theoretical prediction similar to the one described earlier but using optimized scales. The difference as a function of rapidity is shown in figure $5 \mathrm{~b}$. 


\section{Conclusion}

The availability of both proton and antiproton circulating beams in the S̄ppS collider provided a unique opportunity to measure direct photon production in $\overline{\mathrm{p} p}$ and pp interactions at $\sqrt{s}=24.3 \mathrm{GeV}$ with comparable precision. The cross sections presented here clearly demonstrate the importance of the $\bar{q} q$ annihilation term in $\bar{p}$ p direct photon production. No significant differences between $\bar{p} p$ and pp collisions are observed in inclusive $\pi^{0}$ or $\eta$ production.

\section{Acknowledgement}

We wish to thank the CERN PS and SPS Divisions for their magnificent achievement in operating the antiproton source and the $\mathrm{S} \overline{\mathrm{p}} \mathrm{pS}$ collider. We also thank the EP and SPS Divisions for their extensive support during the running of the experiment. We would also like to thank the earlier members of the UA6 Collaboration for their support in the initial stages of the experiment. We gratefully acknowledge the dedicated support of the UA6 technical and secretarial staff. We acknowledge financial support from the following funding agencies: Swiss Science Foundation, and US Department of Energy and National Science Foundation.

\section{References}

[1] W. Vogelsang and M.R.Whalley, J. Phys. G : Nucl. Part. Phys. 23 (1997) Suppl. $7 \mathrm{~A}, \mathrm{~A} 1-\mathrm{A} 69$.

[2] G. Sozzi et al., Phys. Lett. B 317 (1993) 243.

[3] G. Ballocchi et al., Phys. Lett. B 317 (1993) 250.

[4] L. Dick and W. Kubischta, Physics with jet targets at the SPS $\bar{p} p$ collider, in Hadronic physics at intermediate energies, eds. T.Bressani and R.A. Ricci (Elsevier Science Publishers, Amsterdam 1986) p.209.

[5] L. Camilleri et al., Nucl. Instrum. and Methods. A 286 (1990) 49.

[6] G.R. Snow, A Novel Triggering System for the UA6 Electromagnetic Calorimeter, proceedings of The First International Conference on Calorimetry in High Energy Physics, 29 Oct. - 1 Nov. 1990, eds. D. Anderson et al., World Scientific Publishing Co.

[7] GEANT, CERN Program Library Long Writeup W5013 (1994).

[8] T. Sjöstrand, Pythia 5.7 and Jetset 7.4, CERN-TH.7112/93 (1993). 
[9] P. Aurenche et al. Nuclear Physics B 297 (1988) 661.

[10] P. Aurenche et al., Nuclear Physics B 399 (1993) 34.

[11] L.Bourhis et al., Eur. Phys. J. C 2 (1998) 529.

[12] P. Aurenche et al., Physical Review D 39 (1989) 3275. 